\title{
MISCELANEA
}

PRIMERAS JORNADAS CIENTÍFICAS DE ESTUDIANTES DE ENFERMERÍA. FACULTAD CIENCIAS DE LA SALUD. UNIVERSITAT JAUME I. CASTELLÓN DE LA PLANA.

\section{HIGIENE DE MANOS EN EL PERSONAL SANITARIO}

Autores: Carmona Fortuño I; Babiano Gil M; Morales Bacas E; Soriano Martínez C; Esteve Clavero A.

Correo electrónico: Irenecarmona @hotmail.com

Institución: Universitat Jaume I.

Tipo de comunicación: oral.

Introducción: La OMS considera la higiene de manos como la principal medida para reducir las infecciones asociadas a la atención sanitaria, por lo que la promueve como prioritaria dentro de sus iniciativas para mejorar la seguridad de los pacientes. 
Objetivo: Basándonos en esta definición, realizaremos un estudio sobre el conocimiento que tienen los estudiantes de la Universidad Jaume I, de Enfermería y Medicina, acerca del lavado de manos. Esta cuestión es muy importante para la prevención de infecciones nosocomiales ya que la correcta higiene de manos es la principal medida que el personal sanitario puede tomar para evitar la transmisión de enfermedades infecciosas en la práctica clínica, tanto del profesional al paciente como del paciente a éste.

Material y método: La recogida de datos se realizará mediante un cuestionario autoadministrado a alumnos de $1^{\circ}$ y $2^{\circ}$ curso de los grados de Enfermería y Medicina. Posteriormente se realizará un análisis estadístico de los mismos.

Resultados: Los resultados obtenidos, permitirán evaluar los conocimientos previos del alumnado sobre la importancia de la higiene de manos en su futura profesión, los conocimientos en relación a las diferentes técnicas y los factores que la dificultan.

Además, esperamos poder concluir si los conocimientos sobre este tema son suficientes o si es necesario mejorar la educación acerca de la higiene de manos durante su formación universitaria. 